Proceedings of the 1997 IEEE

International Conference on Robotics and Automation

Albuquerque, New Mexico - April 1997

\title{
Target Identification with Multiple Logical Sonars using Evidential Reasoning and Simple Majority Voting *
}

\author{
Birsel Ayrulu ${ }^{1}$, Billur Barshan ${ }^{1}$ and Simukai W. Utete ${ }^{2}$ \\ ${ }^{1}$ Department of Electrical Engineering \\ Bilkent University \\ Bilkent, 06533 Ankara, Turkey \\ ${ }^{2}$ Robotics Research Group \\ Department of Engineering Science \\ University of Oxford \\ Oxford, OX1 3PJ, U.K.
}

\begin{abstract}
In this study, physical models are used to model reflections from target primitives commonly encountered in a mobile robot's environment. These targets are differentiated by employing a multi-transducer pulse/echo system which relies on both amplitude and time-offlight data, allowing more robust differentiation. Target features are generated as being evidentially tied to degrees of belief which are subsequently fused by employing multiple logical sonars at different geographical sites. Feature data from multiple logical sensors are fused with Dempster-Shafer rule of combination to improve the performance of classification by reducing perception uncertainty. Dempster-Shafer fusion results are contrasted with the results of combination of sensor beliefs through simple majority vote. The method is verified by experiments with a real sonar system. The evidential approach employed here helps to overcome the vulnerability of the echo amplitude to noise and enables the modeling of non-parametric uncertainty in real time.
\end{abstract}

\section{Introduction}

One mode of sensing which is potentially very useful and cost-effective for mobile robot applications is sonar. Since acoustic sensors are light, robust and inexpensive devices, they are widely used in applications such as navigation of autonomous vehicles through unstructured environments, map-building [1], target-

*This research is supported by TÜBITAK under grant EEEAG-92 and by NATO collaborative research grant CRG 951262. tracking [2] and obstacle avoidance [3]. Sensory information from a single sonar has poor angular resolution and is not sufficient to differentiate the most commonly encountered target primitives [4]. The most popular sonar ranging system is based on the time-offlight (TOF) measurement which is the time elapsed between the transmission of a pulse and its reception. Since the amplitude of sonar signals is very sensitive to environmental conditions and since standard electronics for the Polaroid sensor [5] do not provide the echo amplitude directly, most sonar systems exploit only TOF information. Differential TOF models of targets have been used by several researchers in mapbuilding, robot localization and target tracking applications: In [6], using a single mobile sensor for map building, edges are differentiated from planes and corners from a single location. Planes and corners are differentiated by scanning from two separate locations using TOF information from complete sonar scans of the targets. In [1], a similar approach has been proposed to identify these targets as beacons for mobile robot localization. Manyika has used differential TOF models for target tracking [7].

For improved target classification, multi-transducer pulse/echo systems which rely on both amplitude and TOF information can be employed. In earlier work by Barshan and Kuc, a methodology based on 'TOF and amplitude information is introduced to differentiate planes and corners [4]. Here, we extend this work and fuse the decisions of multiple sensing agents at distinct geographical sites using belief functions. The ultrasonic reflection process from commonly encountered target primitives is modeled such that sonar pairs became evidential logical sensors. Logical sen- 
sors, as opposed to physical sensors that simply acquire data, process real sensory data in order to generate perception units which are context-dependent interpretations of actual data. An automated perception system for mobile robots fusing uncertain sensory information must be reliable in the sense that it is predictable. Therefore quantitative approaches to uncertainty are needed. These considerations favor measure-based methods of handling sensory data (both physical and logical) at different levels of granularity related to the resolution of the data as well as the time constants of the different sensors. This desire motivates our attempt to abstract the sensor integration problem in a conceptual model where uncertainty about evidence and knowledge can be measured and systematically reduced.

Section 2 explains the sensing configuration used in this study and introduces the target primitives. In Section 3, beliefs are assigned to these target primitives based on both TOF and amplitude characteristics of the data. A description of feature fusion is included when multiple sensing sites are used. Consensus of multiple sensors at these sites is obtained by using the Dempster-Shafer rule of combination. In Section 4, the methodology is verified experimentally in an uncluttered rectangular room where the feature fusion process is demonstrated by employing one to fifteen sensing sites. The results of Dempster-Shafer fusion are also contrasted with those arising when the sensors combine beliefs by simple majority vote. In the last section, concluding remarks are made and directions for future research are motivated.

\section{Sonar Sensing}

The most popular sonar ranging system is the TOF system. In this system, an echo is produced when the transmitted pulse encounters an object and a range value $r$ is produced when the echo amplitude waveform first exceeds a preset threshold level $\tau$ :

$$
r=\frac{c t_{0}}{2}
$$

Here $t_{o}$ is the TOF of the echo signal at which the echo amplitude first exceeds the threshold level and $c$ is the speed of sound in air $(c=343.3 \mathrm{~m} / \mathrm{s}$ at room temperature).

In this study, the far-field model of a piston type transducer having a circular aperture is used [8]. The amplitude of the echo decreases with the inclination angle $\theta$, which is the deviation angle from normal incidence as illustrated in Figure 1. The echo amplitude falls below the threshold level when $\theta>\theta_{0}$ where $\theta_{0}$ is the beam angle which depends on the aperture size

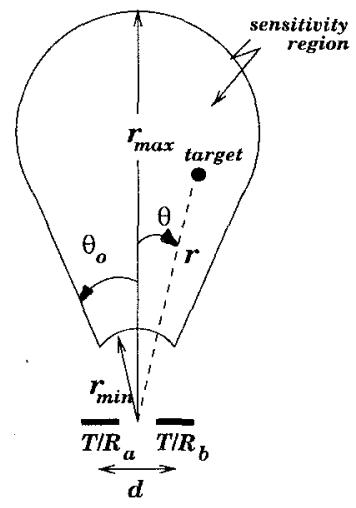

Figure 1: Sensitivity region of an ultrasonic transducer.

and the resonant frequency of the transducer by:

$$
\theta_{0}=\sin ^{-1}\left(\frac{0.61 c}{a f_{0}}\right)
$$

Here $a$ is the transducer aperture radius and $f_{0}$ is the resonant frequency of the transducer.

With a single transducer, it is not possible to estimate the azimuth of a target with better resolution. than the angular resolution of sonar which is approximately $2 \theta_{o}$. In our system, two identical acoustic transducers $a$ and $b$ with center-to-center separation $d$ are employed to improve the angular resolution. Each transducer can operate both as transmitter and receiver. The typical shape of the sensitivity region of the ultrasonic transducer pair is shown in Figure 1. The extent of this region is in general different for each target type since geometrically or physically different targets, in general, exhibit different reflection properties.

In this study, the target primitives modeled are plane, corner and acute corner whose horizontal crosssections are illustrated in Figure 2. Since the wavelength of our sonar $(\lambda \cong 8.6 \mathrm{~mm}$ at $40.0 \mathrm{kHz}$ ) is much larger than the typical roughness of object surfaces encountered in laboratory environments, targets in these environments reflect acoustic beams specularly like a mirror. Hence, while modeling the received signals from these targets, all reflections are considered to be specular which allows transducers both transmitting and receiving to be viewed as a separate transmitter $T$ and virtual receiver $R$ in all cases [9].

Detailed physical reflection models of these target primitives with corresponding echo signal models are provided in [10]. 


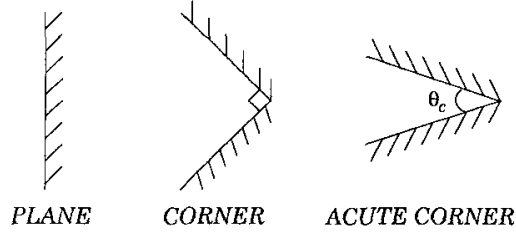

Figure 2: Target primitives modeled and differentiated in this study.

\section{Logical Sensing and Feature Fusion from Multiple Sonars}

This section focuses on the development of a logical sensing module that produces evidential information from uncertain and partial information obtained by multiple sonars at geographically different sensing sites. The formation of such evidential information is accomplished using the theory of belief functions. Belief values are generated by each logical sensor and assigned to the detected features. These features and their evidential metric obtained from multiple sonars are then fused using the Dempster-Shafer rule of combination.

A belief function is a mapping from a class of sets to the interval $[0,1]$ that assigns numerical degrees of support based on evidence [11]. This is a generalization of probabilistic approaches since one is allowed to model ignorance about a given situation. Unlike probability theory, a belief function brings a metric to the intuitive idea that a portion of one's belief can be committed to a set but need not be also committed to its complement. In the target classification problem, ignorance corresponds to not having any information on the type of target that the transducer pair is scanning. Dempster-Shafer theory differs from the Bayesian approach by allowing support for more than one proposition at a time, allowing lack of data (ignorance) to be represented. With this approach, full description of conditional (or prior) probabilities are no longer required and incremental evidence can be easily incorporated. Several researchers have recently been using evidential reasoning in applications such as landmark-based navigation [12] and map-building [13].

To differentiate the target primitives, differences in the reflection characteristics of these targets are exploited and formulated in terms of basic probability masses. This logical sensor model of sonar perception is novel in the sense that it models the uncertainties associated with the target type. The uncertainty in the measurements of each sonar pair is represented by a belief function having target type or feature as a focal element with basic probability mass $m($.$) associ-$ ated with this feature:

$$
B F=\{\text { feature } ;(\text { feature })\}
$$

Logical sensing of the target primitives is accomplished through a metric as degrees of belief assigned to plane, corner and acute corner according to the amplitude and TOF characteristics of the received signals from these target primitives. The differentiation algorithm is basically an extension of the algorithm in [4] and is detailed in [10]. Here, we focus on the basic probability assignment to each feature and the feature fusion process:

$$
\begin{aligned}
& m(p)=\left(1-I_{4}\right) I_{1} \frac{\left[A_{a a}(\theta)-A_{a b}(\theta)\right]\left[A_{b b}(\theta)-A_{a b}(\theta)\right]}{\max \left[A_{a a}(\theta)-A_{a b}(\theta)\right] \max \left[A_{b b}(\theta)-A_{a b}(\theta)\right]} \\
& m(c)=\left(1-I_{4}\right) \frac{I_{2}\left[A_{a b}(\theta)-A_{a a}(\theta)\right]+I_{3}\left[A_{a b}(\theta)-A_{b b}(\theta)\right]}{I_{2} \max \left[A_{a b}(\theta)-A_{a a}(\theta)\right]+I_{3} \max \left[A_{a b}(\theta)-A_{b b}(\theta)\right]} \\
& \quad \text { if } I_{2} \neq 0 \text { or } I_{3} \neq 0 \\
& \quad \text { else } 0 \\
& m(a c)=I_{4} \frac{\left[t_{a a}(\theta)-t_{a b}(\theta)\right]\left[t_{b b}(\theta)-t_{a b}(\theta)\right]}{\max \left\{\left[t_{a a}(\theta)-t_{a b}(\theta)\right]\left[t_{b b}(\theta)-t_{a b}(\theta)\right]\right\}}
\end{aligned}
$$

where $A_{a b}(\theta)$ denotes maximum value of $A_{a b}(r, \theta, d, t)$ which is the signal transmitted by transmitter $b$ and received by receiver $a$, and $t_{a b}(\theta)$ denotes TOF extracted from $A_{a b}(r, \theta, d, t)$ at angle $\theta$ by thresholding. Definitions of $A_{a a}(\theta)$ and $A_{b b}(\theta)$ are similar. $I_{1}, I_{2}, I_{3}$ and $I_{4}$ are the indicators of the conditions given below:

$$
\begin{aligned}
& I_{1}= \begin{cases}1 & \text { if }\left[A_{a a}(\theta)-A_{a b}(\theta)\right]>\sigma_{A} \text { and }\left[A_{b b}(\theta)-A_{a b}(\theta)\right]>\sigma_{A} \\
0 & \text { otherwise }\end{cases} \\
& I_{2}= \begin{cases}1 & \text { if }\left[A_{a b}(\theta)-A_{a a}(\theta)\right]>\sigma_{A} \\
0 & \text { otherwise }\end{cases} \\
& I_{3}= \begin{cases}1 & \text { if }\left[A_{a b}(\theta)-A_{b b}(\theta)\right]>\sigma_{A} \\
0 & \text { otherwise }\end{cases} \\
& I_{4}= \begin{cases}1 & \text { if }\left[t_{a a}(\theta)-t_{a b}(\theta)\right]>\sigma_{t} \text { and }\left[t_{b b}(\theta)-t_{a b}(\theta)\right]>\sigma_{t} \\
0 & \text { otherwise }\end{cases}
\end{aligned}
$$

Remaining belief is assigned to an unknown target type, representing ignorance or undistributed probability mass, as:

$$
m(u)=1-[m(p)+m(c)+m(a c)]
$$

For the Dempster-Shafer rule of combination to be applicable, the sources of information to be fused must be independent [11]. This is the case in our application. Given two sources with belief functions,

$$
\begin{aligned}
& B F_{1}=\left\{f_{i}, m\left(f_{i}\right)\right\}_{i=1}^{4}=\{p, c, a c, u ; m(p), m(c), m(a c), m(u)\} \\
& B F_{2}=\left\{g_{j}, m\left(g_{j}\right)\right\}_{j=1}^{4}=\{p, c, a c, u ; m(p), m(c), m(a c), m(u)\}
\end{aligned}
$$

consensus is obtained as the orthogonal sum:

$$
\begin{aligned}
B F & =B F_{1} \oplus B F_{2} \\
& =\left\{h_{k}, m\left(h_{k}\right)\right\}_{k=1}^{4}=\left\{p, c, a c, u ; m_{c}(p), m_{c}(c), m_{c}(a c), m_{c}(u)\{8)\right.
\end{aligned}
$$

which is both associative and commutative with the resulting operation being shown in Table 1 . The sequential combination of multiple bodies of evidence can be obtained for $n$ sensor pairs as:

$$
B F=\left(\left(\left(B F_{1} \oplus B F_{2}\right) \oplus B F_{3}\right) \ldots \oplus B F_{n}\right)
$$




\begin{tabular}{|c||c|c|c|c|}
\hline$B F_{1}$ & $\begin{array}{c}\text { plane } \\
m_{1}(p)\end{array}$ & $\begin{array}{c}\text { corner } \\
m_{1}(c)\end{array}$ & $\begin{array}{c}\text { acute corner } \\
m_{1}(a c)\end{array}$ & $\begin{array}{c}\text { unknown } \\
m_{1}(u)\end{array}$ \\
\hline \hline plane & plane & $\varnothing$ & $\varnothing$ & plane \\
$m_{2}(p)$ & $m_{1}(p) m_{2}(p)$ & $m_{1}(c) m_{2}(p)$ & $m_{1}(a c) m_{2}(p)$ & $m_{1}(u) m_{2}(p)$ \\
\hline corner & $\emptyset$ & corner & $\emptyset$ & corner \\
$m_{2}(c)$ & $m_{1}(p) m_{2}(c)$ & $m_{1}(c) m_{2}(c)$ & $m_{1}(a c) m_{2}(c)$ & $m_{1}(u) m_{2}(c)$ \\
\hline acute corner & $\varnothing$ & $\emptyset$ & acute corner & acute corner \\
$m_{2}(a c)$ & $m_{1}(p) m_{2}(a c)$ & $m_{1}(c) m_{2}(a c)$ & $m_{1}(a c) m_{2}(a c)$ & $m_{1}(u) m_{2}(a c)$ \\
\hline unknown & plane & corner & acutecorner & unknown \\
$m_{2}(u)$ & $m_{1}(p) m_{2}(u)$ & $m_{1}(c) m_{2}(u)$ & $m_{1}(a c) m_{2}(u)$ & $m_{1}(u) m_{2}(u)$ \\
\hline
\end{tabular}

Table 1: Target differentiation by Dempster-Shafer rule of combination.

Using the Dempster-Shafer rule of combination:

$$
m\left(h_{k}\right)=\frac{\sum \sum_{h_{k}=f_{i} \cap g_{j}} m\left(f_{i}\right) m\left(g_{j}\right)}{1-\sum \sum_{h_{k}=f_{i} \cap g_{j}=\varnothing} m\left(f_{i}\right) m\left(g_{j}\right)}
$$

where $\sum \sum_{h_{k}=f_{i} \cap g_{j}=\emptyset} m\left(f_{i}\right) m\left(g_{j}\right)$ is a measure of conflict. The consensus belief function representing the feature fusion process has the metrics

$$
\begin{aligned}
m(p) & =\frac{m_{1}(p) m_{2}(p)+m_{1}(p) m_{2}(u)+m_{1}(u) m_{2}(p)}{1-\text { conflict }} \\
m(c) & =\frac{m_{1}(c) m_{2}(c)+m_{1}(c) m_{2}(u)+m_{1}(u) m_{2}(c)}{1-\text { conflict }} \\
m(a c) & =\frac{m_{1}(a c) m_{2}(a c)+m_{1}(a c) m_{2}(u)+m_{1}(u) m_{2}(a c)}{1-\text { conflict }} \\
m(u) & =\frac{m_{1}(u) m_{2}(u)}{1-\text { conflict }}
\end{aligned}
$$

In the above equations, the term "conflict" represents the disagreement in the consensus of two logical sensing units, thus representing the degree of mismatch in the fusion of features perceived at two different sonar sites. The metric evaluating conflict is expressed as:

$$
\begin{aligned}
\text { conflict } & =m_{1}(p) m_{2}(c)+m_{1}(c) m_{2}(p)+m_{1}(c) m_{2}(a c) \\
& +m_{1}(a c) m_{2}(c)+m_{1}(a c) m_{2}(p)+m_{1}(p) m_{2}(a c)
\end{aligned}
$$

The beliefs are then rescaled after discounting this conflict and may be used in further data fusion processes.

\section{Experimental Verification}

In this study, an experimental set-up is employed to assign belief values to target type based on experimentally obtained TOF and amplitude characteristics of the target primitives, and to test the proposed fusion method for target classification. Panasonic transducers are used with aperture radius $a=0.65 \mathrm{~cm}$ and resonant frequency $f_{0}=40 \mathrm{kHz}$, therefore $\theta_{0} \cong 54^{\circ}$ for these transducers. These transducers are manufactured with distinct characteristics for transmitting and receiving; two pairs of vertically very closely spaced transmitter and receiver, illustrated in Figure 3, are

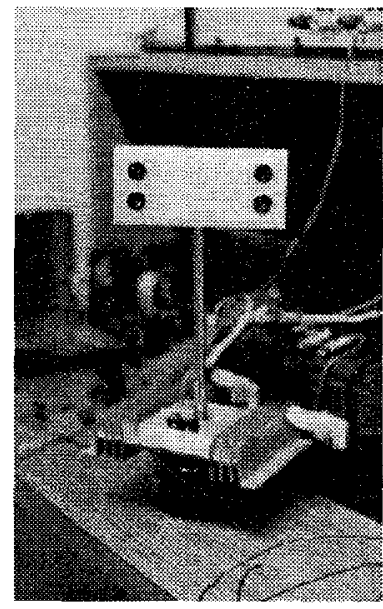

Figure 3: The logical sensing unit.

used as a single logical sensing unit. The horizontal center-to-center separation between the transducers is $d=24 \mathrm{~cm}$. This sensing unit is mounted on a small $6 \mathrm{~V}$ stepper motor with step size $0.9^{\circ}$. The motion of the stepper motor is controlled by the parallel port of an IBM-PC 486 and the aid of a microswitch. Data acquisition from the sonars is accomplished by using a DAS-50 A/D card with 12-bit resolution and $1 \mathrm{MHz}$ sampling frequency. Echo signals are processed on an IBM-PC 486 using the C language. Starting at the transmit time, 10,000 samples of each echo signal have been collected and thresholded. The amplitude information is extracted by finding the maximum value of the signal after the threshold value is exceeded.

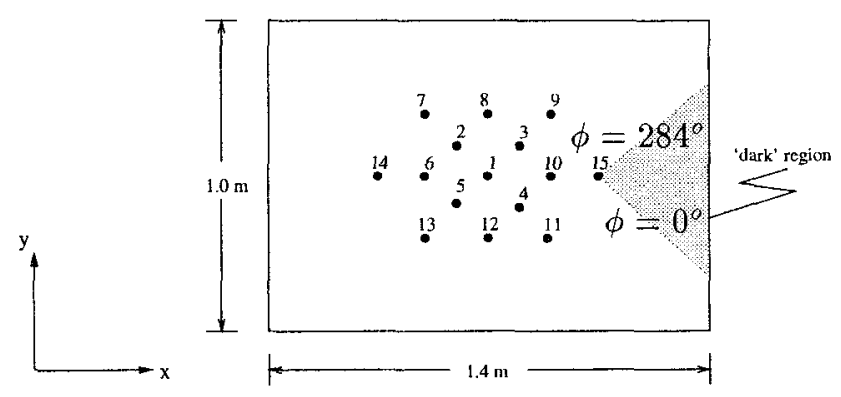

Figure 4: The fifteen sensing sites in the rectangular room.

The method is tested experimentally in an uncluttered rectangular room measuring $1.0 \mathrm{~m}$ by $1.4 \mathrm{~m}$ with specularly reflecting surfaces. The room is scanned by sensing units located at the fifteen positions shown in Figure 4 . The range readings of the transducer pair 2 located at $(-10 \mathrm{~cm}, 10 \mathrm{~cm})$ are given in Figure 5 as an example. Due to the physical limitations of the hard- 


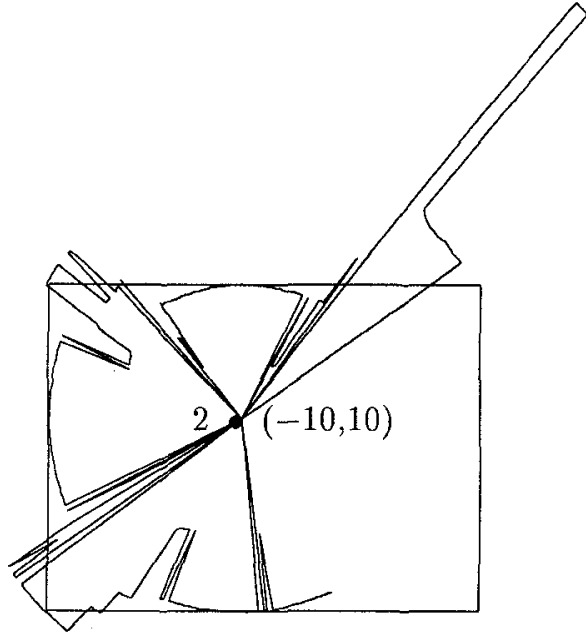

Figure 5: Range readings of the sensor unit 2 located at $(-10 \mathrm{~cm}, 10 \mathrm{~cm})$ in the rectangular room.

ware, the sensors cannot cover the whole range of $\phi$ but rotate over the range $0^{\circ} \leq \phi \leq 284^{\circ}$.

Feature beliefs are assigned by the sensors based on the TOF and amplitude characteristics of the sonar signals reflected from corners and planar walls. Examples of basic probability assignments by individual sensors are shown in Figure 6. Note the high degree of uncertainty since a single logical sensor is employed. Each of the sensor decisions on target type is referred to the central position for comparison and fusion. During a scan, a sensor estimates the range and angle of the target under observation. The values for a target are weighted by the beliefs assigned to the estimates and then referred to position $(0,0)$. The sensors' determinations of beliefs are fused using the DempsterShafer rule of combination. Results are shown in Figure $7(\mathrm{a})$.

The sensors' beliefs about target type were also combined using simple majority voting. The beliefs about target type were counted as votes and the majority vote taken as the outcome. Once again, the weighted averages were computed and referred to the central location. The corresponding results are shown in Figure 7(b). In the room experiment, conflicts over target type are primarily the result of noisy amplitude signals when the target is visible. Combination by voting provides a means of resolving target type in cases of conflict.

To show the accumulation of evidence, plots of correct decision percentage as a function of number of sensor pairs used are given in Figure 8 for both methods of fusion. In both the case of Dempster-Shafer fusion and that of simple majority vote, the sensors arrived at the correct decisions on target type for all targets. However, the maximum percentage of correct

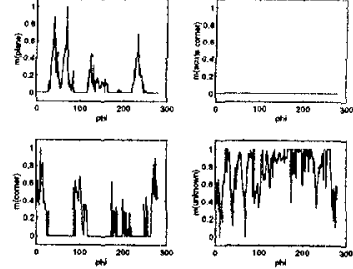

(a)

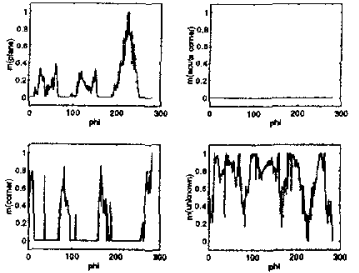

(b)
Figure 6: Belief assignment by the sensors located at (a) $(0 \mathrm{~cm}, 0 \mathrm{~cm})(\mathrm{b})(-10 \mathrm{~cm}, 10 \mathrm{~cm})$.
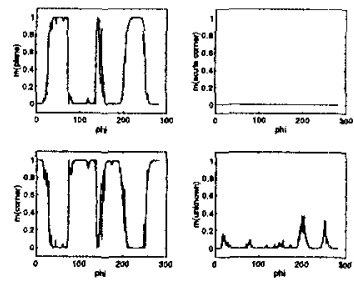

(a)
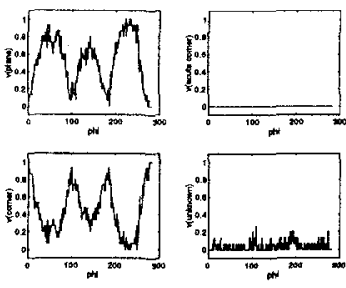

(b)
Figure 7: Results of (a) Dempster-Shafer rule (b) simple voting algorithm.

decisions achievable is below $100 \%$ because at certain viewpoints during a scan the target may not be visible. Using a single sensor, percentage of correct decisions is about $30 \%$. The remaining $70 \%$ is attributed to incorrect decisions due to noise and complete uncertainty which occurs when the target is not visible to the sensor. When decisions of fifteen pairs are fused using the Dempster-Shafer method, correct decision percentage improves to $61.1 \%$. With simple majority voting, the corresponding number is $70.4 \%$. Note that after simple-voting fusion from about five pairs, the correct decision percentage remains approximately constant around $70 \%$, indicating redundancy in the number of sensors employed.

\section{Conclusion}

This work presents a novel application of the theory of evidence for target (beacon) recognition. Physical models are used to model reflections from target primitives commonly encountered in mobile robot applications. Target features are generated as being evidentially tied to degrees of belief which are subsequently fused for multiple sonars at distinct geographical sites. Using both TOF and amplitude data in the feature fusion process allows more robust differentiation. The belief function approach is contrasted with combination of sensor beliefs by simple majority vote. For the simple targets in our room, vot- 


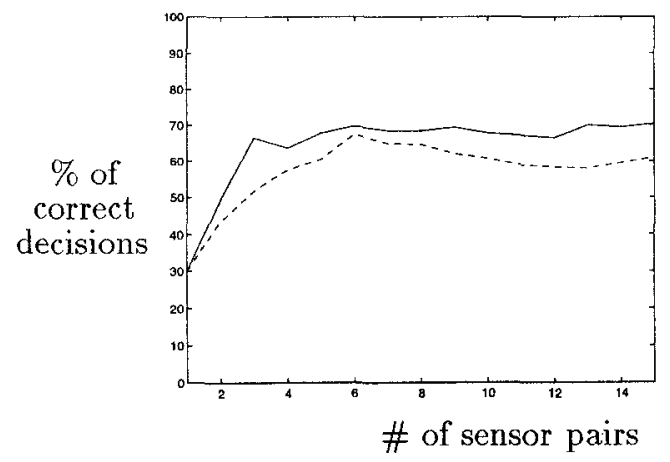

Figure 8: Correct decision percentage of DempsterShafer rule (dashed line) and simple voting algorithm (solid line) versus number of sensors employed in the fusion process.

ing achieves a known and correct target decision in all cases, resolving conflicts through the taking of the majority decision. The belief function approach employed in the differentiation of the target primitives enables the modeling of non-parametric uncertainty. Fusion of feature data from multiple sensors using Dempster-Shafer rule of combination reduces such perception uncertainty. Although there is a consequent increase in processing time, this is insignificant considering the fast processing speeds of modern computers. It has been experimentally demonstrated that the belief function methodology is suitable for real-time applications when multiple sensing sites are used. The results have ground for application in mobile robotics where multiple sensing agents or robots are employed to survey an unknown environment composed of primitive target types. As for future work, the proposed fusion method can be extended to include physically different sensors such as infrared and laser-ranging systems for map-building, target identification, localization and tracking applications. Coordination of the sensing agents and strategic target recognition while either or both the sensors and the targets are in motion is another possible direction for future research. Future work could also look at more complex voting strategies and the situation where sensors are nonequal voters or coalitions are formed.

\section{References}

[1] J. J. Leonard and H. F. Durrant-Whyte. Directed Sonar Navigation. Kluwer Academic Press, London, 1992 .

[2] R. Kuc, "Three-dimensional tracking using qualitative bionic sonar," Robotics and Autonomous
Systems, vol. 11, pp. 213-219, 1993.

[3] J. Borenstein and Y. Koren, "Obstacle avoidance with ultrasonic sensors," IEEE Transactions on Robotics and Automation, vol. RA-4, pp. 213-218, April 1988.

[4] B. Barshan and R. Kuc, "Differentiating sonar reflections from corners and planes by employing an intelligent sensor," IEEE Transactions on Pattern Analysis and Machine Intelligence, vol. 12, pp. 560-569, June 1990.

[5] Polaroid Corporation. "Ultrasonic components group," 119 Windsor St., Cambridge, MA 02139, 1990.

[6] Ö. I. Bozma. A Physical Model-Based Approach to Analysis of Environments using Sonar. $\mathrm{PhD}$ thesis, Yale University, New Haven, CT, May 1992.

[7] J. Manyika and H. F. Durrant-Whyte. Data Fusion and Sensor Management: A Decentralized Information-Theoretic Approach. Ellis Horwood, New York, 1994.

[8] J. Zemanek, "Beam behaviour within the nearfield of a vibrating piston," The Journal of the Acoustical Society of America, vol. 49, pp. 181-191, January 1971.

[9] R. Kuc and M. W. Siegel, "Physically-based simulation model for acoustic sensor robot navigation," IEEE Transactions on Pattern Analysis and Machine Intelligence, vol. PAMI-9, pp. 766778, November 1987.

[10] B. Ayrulu. "Classification of target primitives with sonar using two non-parametric data fusion methods," Master's thesis, Bilkent University, Ankara, Turkey, July 1996.

[11] G. Shafer. A Mathematical Theory of Evidence. Princeton:Princeton University Press, 1976.

[12] R. R. Murphy, "Adaptive rule of combination for observations over time," in Proceedings of the IEEE/SICE/RSJ International Conference on Multisensor Fusion and Integration for Intelligent Systems, pp. 125-131, Washington D.C., December 1996.

[13] D. Pagac, E. M. Nebot and H. F. DurrantWhyte, "An evidential approach to probabilistic map-building," in Proceedings IEEE International Conference on Robotics and Automation, pp. 745-750, Minneapolis, MN, April 1996. 